\title{
Rare and Atypical Presentation of Glomus Tumor in the Eyelid
}

\section{Dear Editor,}

Glomus tumors are rare, benign vascular tumors, arising from the glomus body, an arteriovenous structure with thermoregulatory function. We describe an atypical presentation of a glomus tumor that occurred as a painless nodular eyelid mass.

A 30-year-old male presented with a history of painless swelling in the right lower lid of 6 months duration, which had gradually increased in size (Fig. 1A). Best-corrected visual acuity in both eyes were 20 / 20. A non-tender, firm, nodular bilobed lesion with smooth surface about $3 \mathrm{~mm}$ away from the lid margin was noted in his right eye. No signs of inflammation were present and overlying skin was normal in appearance. It was neither adherent to skin above nor to the structure underneath. Posterior edge of the mass could be felt. Rest of the ocular examination was within normal limits. Left eye was unremarkable on examination. Provisional diagnosis of a solitary benign neurogenic tumor or an implantation dermoid was made. Patient underwent an excision biopsy of the mass.

Intraoperatively, a $9 \times 5 \times 4$-mm subcutaneous, bilobed, pink, non-encapsulated mass was seen (Fig. 1B). Unusually excessive bleeding was encountered during dissection following which the mass was completely excised.

Histopathological examination of the tissue specimen revealed a well circumscribed neoplasm composed of irregularly dilated vessels, surrounded by collars of homogenous tumor cells forming nests (Fig. 1C). Tumor cells were uniform, round to oval with lightly eosinophilic cytoplasm having a centrally placed rounded or punched out nucleus. No atypia or mitoses was noted. The tumor cells were interspersed with smooth muscle cells. Immunohistochemistry further revealed strong cytoplasmic positivity for

Received: December 8, 2020 Final revision: January 31, 2021 Accepted: March 9, 2021

smooth muscle actin (Fig. 1D). These findings were consistent with the diagnosis of glomus cell tumor. Postoperative period was uneventful without any evidence of recurrence for the last 2 years.

Glomus tumors are benign perivascular neoplasms, typically seen in young adults in the $3 \mathrm{rd}$ and 4 th decade. They are known to arise from the glomus body, an arteriovenous anastomosis surrounded by smooth muscle cells. These are commonly seen in distal extremities of the body [1], subungual region of the fingers being the most common site.

Ocular incidences of glomus tumors are rare. There have been very few cases of orbital glomus tumors reported in previous literature most of which presented as an orbital swelling with gradually increasing proptosis [1]. To the best of our knowledge and after a thorough literature search very few cases of these tumors in the eyelids have been reported. The occurrence of glomus tumors in the eyelid is rather rare and it may be suggestive of the presence of glomus bodies in the lid, although this has not been confirmed [2].

In 1941, Kirby [2] reported first case of glomus tumor which presented as an eyelid mass. Cases reported earlier
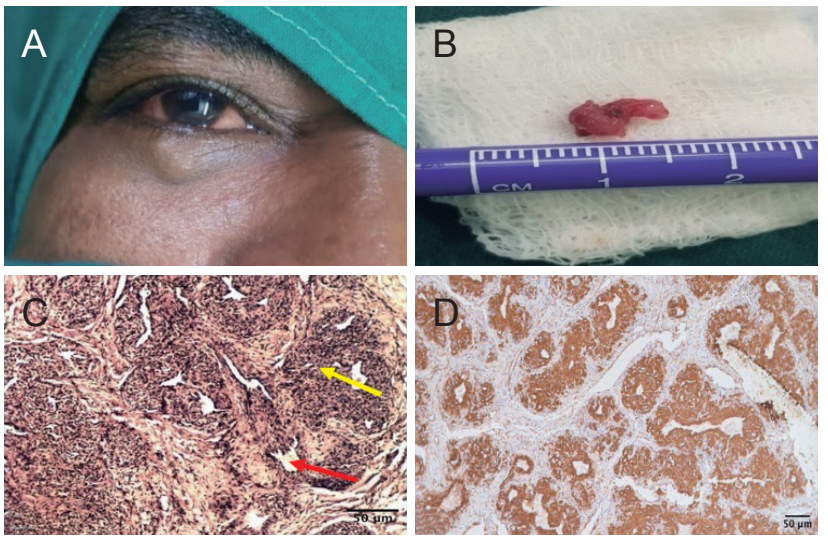

Fig. 1. Clinical, gross, and histopathological images of glomus tumor in the eyelid. (A) Right lower eyelid swelling. (B) Tissue specimen of bilobed, pinkish white, nonencapsulated mass measuring $9 \times 5 \times 4 \mathrm{~mm}$. (C) Histopathological specimen of glomus tumor $(\mathrm{H} \& \mathrm{E}$, low magnification, $\times 10)$ showing irregularly dilated blood vessels (red arrow) surrounded by collars of glomus cells (yellow arrow). (D) Immunohistochemistry showing strong cytoplasmic positivity of tumor cells to smooth muscle actin. Informed consent for publication of the clinical images was obtained from the patient. 
by Jensen [3] and Saxe et al. [4] characteristically described this lesion as a bluish/violaceous soft spongy mass in the eyelid. In another case of glomus tumor reported by Mortada in 1963 [5], a tender firm mass was described. In our case, we came across an eyelid mass with an atypical presentation as a firm swelling with no discoloration. Unlike in the last-mentioned case where the mass was painful, growth in our patient was non tender.

Histopathological findings and immunohistochemistry disproved our provisional diagnosis of a neurofibroma, which is typically composed of spindle cells. Intraoperative bleeding also suggested a vascular tumor thus ruling out earlier diagnoses.

Definitive diagnosis of a glomus tumor requires histopathological examination and immunohistochemistry. Glomus tumors are managed successfully by complete surgical excision [4], with low rate of recurrence. It becomes mandatory for all excised eye lid lesions to be subjected to histopathological evaluation failing which, rare tumors can go undocumented. Therefore, glomus tumors can be considered as one of the differential diagnosis in patients presenting with an eyelid mass.

\section{Neha Panickar, Shruthi Tara V}

Department of Orbit and Oculoplasty, Sankara Eye Hospital, Coimbatore, India

E-mail (Shruthi Tara V): shruthitara@sankaraeye.com

\section{Conflict of Interest}

No potential conflict of interest relevant to this article was reported.

\section{Acknowledgements}

The authors acknowledge and credit Dr. Alamelu Jayaraman and Dr. M. Murthy for their valuable contribution in the histopathological evaluation of tissue in this patient.

\section{References}

1. Chang M, Lee Y, Baek S, Lee TS. Orbital glomus tumor in an Asian patient. BMC Ophthalmol 2012;12:62.

2. Kirby DB. Neuromyoarterial glomus tumor in the eyelid. Trans Am Ophthalmol Soc 1940;38:80-7.

3. Jensen OA. Glomus tumor (glomangioma) of eyelid. Arch Ophthalmol 1965;73:511-3.

4. Saxe SJ, Grossniklaus HE, Wojno TH, et al. Glomus cell tumor of the eyelid. Ophthalmology 1993;100:139-43.

5. Mortada A. Glomangioma of the eyelid. Br J Ophthalmol 1963;47:697-9. 